\title{
Herzlich Willkommen zur VENALPINA IX
}

\section{6.-31.01.2020, Seefeld in Tirol}

Liebe Kolleginnen und Kollegen!

Im kommenden Januar (letzte Woche, 26.31.01.2020) organisieren Prof. Dr. med. Wolfgang Salmhofer (Graz), Dr. Dominic Mühlberger (Bochum) und ich selbst (Jürg Hafner) die VENALPINA IX in Seefeld in Tirol.

Das Programm ist wirklich attraktiv. Die Vorträge werden sowohl von jüngeren als auch von sehr erfahrenen Referentinnen und Referenten der 3 „DACH“-Länder gehalten und mit interaktiven Diskussionen ergänzt. Den roten Faden bilden die „KeyNote-Lectures“:

- So, 26.01.2020, 17.30 Uhr: Michael Jünger (Greifswald): Telemedizin in der Phlebologie

- Mo, 27.01.2020, 11.00 Uhr: Christian Neumann (Wien): May-Thurner-Syndrom

- Mo, 27.01.2020, 17.30 Uhr: Jan Dirk Studt (Zürich): Antikoagulation: Standards, Ausnahmen
- Di, 28.01.2020, 11.00 Uhr: Bernhard Zelger (Innsbruck): Moderne Sicht auf die Vaskulitiden

- Di, 28.01.2020, 17.30 Uhr: Jürg Traber (Kreuzlingen): Pelvines Stauungssyndrom

- Mi, 29.01.2020, 11.00 Uhr: Elena Conde-Montero (Madrid): Chronische Wunden in einem Armenviertel Madrids

- Mi, 29.01.2020, 17.30 Uhr: Catherine van Montfrans (Rotterdam): Interdisziplinäre Phlebologie

- Do, 30.01.2020, 11.00 Uhr: Manfred Baldt (Wolfsberg): Bildgebung in der Phlebologie

- Do, 30.01.2020, 17.30 Uhr: Stephan Wagner (Bad Zurzach): Lymphologie 2020

- Fr, 31.01.2020, 11.00 Uhr: Eberhard Rabe (Bonn): „What's new in Phlebology“

Das Gesamtprogramm deckt die Phlebologie und Lymphologie in ihren internistischen, chirurgischen und dermatologi- schen Aspekten vollumfassend ab und ist sowohl für Assistenzärztinnen und Assistenzärzte in Weiterbildung als auch für erfahrene Phlebologinnen und Phlebologen gleichermaßen geeignet.

Neben dem wissenschaftlichen und klinischen Programm bietet die VENALPINA viel Gelegenheit zum freundschaftlichen Austausch: „Make new friends and keep the old ones." Wir würden uns sehr freuen, wenn Sie alle zur VENALPINA IX nach Seefeld in Tirol reisen könnten und den ganzen Kongress oder mindestens einen Teil davon besuchen würden.

Mit herzlichen und kollegialen Güssen Prof Dr. med. Wolfgang Salmhofer, Dr. med. Dominic Mühlberger, Prof. Dr. med. Jürg Hafner 